\title{
DNA Replication
}

\author{
Tariku Simion* \\ South Agricultura Research Institute, Arbaminch Agricultural Research Center, Ethopia
}

Submission: June 06, 2018; Published: September 18, 2018

*Corresponding author: Tariku Simion, Southern Agricultural Research Institute, Arbaminch Agricultural Research Center, Arba Minch, Ethiopia, Email: trk2011smn@gmail.com

Abstract

Nearly every cell in a person's body has the same DNA. Most DNA is located in the cell nucleus, but a small amount of DNA can also be found in the mitochondria. DNA was thought to be a simple molecule, consisting of nucleotides strung together like beads on a string.By the late 1940s biochemists knew that DNA was a very long polymer made up of millions of nucleotides. DNA is made up of two strands and each strand of the original DNA molecule serves as a template for the production of the complementary strand, a process referred to as semi conservative replication. Cellular proofreading and error-checking mechanisms ensure near perfect fidelity for DNA replication. DNA usually exists as a double-stranded structure, with both strands coiled together to form the characteristic double- helix. Each single strand of DNA is a chain of four types of nucleotides. Nucleotides in DNA contain a deoxyribose sugar, a phosphate, and a nucleobase. The four types of nucleotide correspond to the four-nucleobase adenine; cytosine, guanine, and thymine, commonly abbreviated as A, C, G and T. This review was assumed to have the historical background of DNA replicationand major DNA replication steps and its function.

Keywords: Nucleobase;Double- helix;Nucleus;Complementary and deoxyribose

\section{Introduction}

DNA, or deoxyribonucleic acid, is the hereditary material in humans and almost all other organisms. Nearly every cell in a person's body has the same DNA. Most DNA is located in the cell nucleus (where it is called nuclear DNA), but a small amount of DNA can also be found in the mitochondria (where it is called mitochondrial DNA or mtDNA).DNA has a fascinating history. The Swiss scientist Friedrich Miescher discovered DNA near the end of the nineteenth century. Miescher never knew that the substance he had isolated from sperm and pus (yes, pus!) would turn out to be so critical to the understanding of life. He died several decades before the function of DNA and its famous double-helical structure were uncovered. After Miescher, other scientists tried to identify the chemical composition of sperm, reasoning that sperm must carry the genetic material to the next generation. These scientists also reasoned that sperm cells have very little excess cellular material other than the hereditary material found in the sperm head. In fact, DNA constitutes over 60 percent of the sperm head; the remainder is mostly protein. For a long time after Miescher's discovery, DNA was thought to be a simple molecule, consisting of nucleotides strung together like beads on a string.By the late 1940s biochemists knew that DNA was a very long polymer made up of millions of nucleotides.Each nucleotide is composed of nitrogen containing nucleobase cytosine $(C)$, guanine $(G)$, adenine $(\mathrm{A})$, and thymine $(\mathrm{T})$ as well as a monosaccharide sugar called deoxyribose and a phosphate group. The nucleotides are joined to one another in a chain by covalent bonds between the sugar of one nucleotide and the phosphate of the next, resulting in an alternating sugar-phosphate backbone. According to base pairing rules (A with $\mathrm{T}$, and $\mathrm{C}$ with $\mathrm{G}$ ), hydrogen bonds bind the nitrogenous bases of the two separate polynucleotide strands to make double-stranded DNA [1].

Nucleic acids composed of the three major macromolecules (sugar, nitrogen base and phosphate groups) are essential for all known forms of life. Most DNA molecules consist of two biopolymer strands coiled around each other to form a double helix. The two DNA strands are known as polynucleotide, since they are composed of simpler units called nucleotides [1].The objectives of this paper are to review the historical background of DNA replicationand major DNA replication steps and its function.

\section{DNA Structure}

DNA usually exists as a double-stranded structure, with both strands coiled together to form the characteristic double- helix. Each single strand of DNA is a chain of four types of nucleotides. Nucleotides in DNA contain a deoxyribose sugar, a phosphate, and a nucleobase. The four types of nucleotide correspond to the fournucleobase adenine; cytosine, guanine, and thymine, commonly abbreviated as A, C, G and T. Adenine and guanine are purine bases, while cytosine and thymine are pyrimidines. These nucleotides form phosphodiester bonds, creating the phosphate-deoxyribose 
backbone of the DNA double helix with the nucleobase pointing inward. Nucleotides (bases) are matched between strands through hydrogen bonds to form base pairs. Adenine pairs with thymine (two hydrogen bonds), and guanine pairs with cytosine (stronger: three hydrogen bonds).

DNA strands have directionality and the different ends of a single strand are called the " 3 ' (three-prime) end" and the " 5 ' (five-prime) end". By convention, if the base sequence of a single strand of DNA is given, the left end of the sequence is the 5' end, while the right end of the sequence is the $3^{\prime}$ end. The strands of the double helix are anti-parallel with one being $5^{\prime}$ to 3 ', and the opposite strand 3' to 5 '. These terms refer to the carbon atom in deoxyribose to which the next phosphate in the chain attaches. Directionality has consequences in DNA synthesis, because DNA polymerase can synthesize DNA in only one direction by adding nucleotides to the 3' end of a DNA strand.

\section{DNA Replication}

DNA replication is the process of producing two identical replicas from one original DNA molecule. This biological process occurs in all living organisms and is the basis for biological inheritance. DNA is made up of two strands and each strand of the original DNA molecule serves as a template for the production of the complementary strand, a process referred to as semi conservative replication. Cellular proofreading and error-checking mechanisms ensure near perfect fidelity for DNA replication [2] (Figure 1).According to Berg et al. [2] in a cell, DNA replication begins at specific locations, or origins of replication, in the genome.

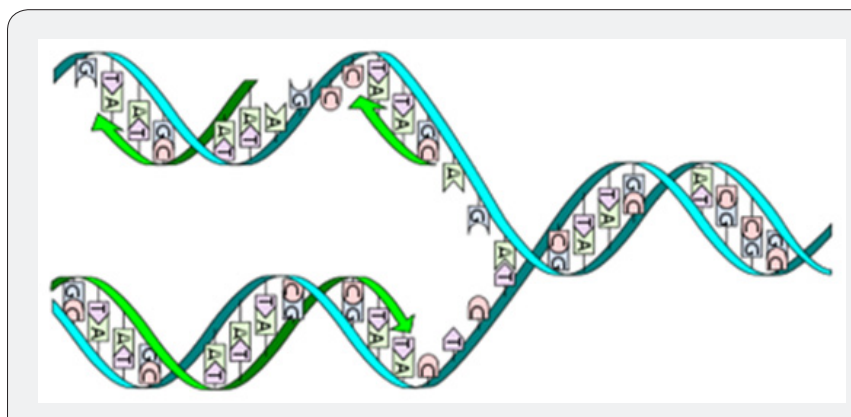

Figure 1: DNA Replication Process.

Unwinding of DNA at the origin and synthesis of new strands results in replication forks growing bidirectional from the origin. A number of proteins are associated with the replication fork which helps in terms of the initiation and continuation of DNA synthesis. Most prominently, DNA polymerase synthesizes the new DNA by adding complementary nucleotides to the template strand.

DNA replication can also be performed in vitro (artificially, outside a cell). DNA polymerases isolated from cells and artificial DNA primers can be used to initiate DNA synthesis at known sequences in a template DNA molecule. The polymerase chain reaction (PCR), a common laboratory technique, cyclically applies such artificial synthesis to amplify a specific target DNA fragment from a pool of DNA.

\section{Replication Process or Steps in DNA Replication}

The pairing of complementary bases in DNA through hydrogen bonding means that the information contained within each strand is redundant. The nucleotides on a single strand can be used to reconstruct nucleotides on a newly synthesized partner strand [2].

\section{Initiation}

For a cell to divide, it must first replicate its DNA[3].This process is initiated at particular points in the DNA, known as "origins", which are targeted by initiator proteins [4]. In E. coli this protein is DNA; in yeast, this is the origin recognition complex [5].Sequences used by initiator proteins tend to be "AT-rich" (rich in adenine and thymine bases), because A-T base pairs have two hydrogen bonds (rather than the three formed in a C-G pair) which are easier to unzip [6].Once the origin has been located, these initiators recruit other proteins and form the pre-replication complex, which unzips the double-stranded DNA.

\section{Elongation}

DNA polymerase has 5'-3' activity. All known DNA replication systems require a free 3 ' hydroxyl group before synthesis can be initiated (Important note: DNA is read in 3' to 5' direction where as a new strand is synthesized in the $5^{\prime}$ to $3^{\prime}$ direction-this is often confused). Four distinct mechanisms for initiation of synthesis are recognized. These are

i. All cellular life forms and many DNA viruses, phages and plasmids use a primase to synthesize a short RNA primer with a free 3' OH group which is subsequently elongated by a DNA polymerase.

ii. The retro elements (including retroviruses) employ a transfer RNA that primes DNA replication by providing a free $3^{\prime} \mathrm{OH}$ that is used for elongation by the reverse transcriptase.

iii. In the adenoviruses and the $\varphi 29$ family of bacteriophages, the 3' $\mathrm{OH}$ group is provided by the side chain of an amino acid of the genome attached protein (the terminal protein) to which nucleotides are added by the DNA polymerase to form a new strand.

iv. In the single stranded DNA viruses- a group that includes the circo viruses, the geminiviruses, the parvoviruses and others and the many phages and plasmids that use the rolling circle replication (RCR) mechanism, the RCR endonuclease creates a nick in the genome strand (single stranded viruses) or one of the DNA strands (plasmids). The $5^{\prime}$ end of the nicked strand is transferred to a tyrosine residue on the nuclease and the free $3^{\prime} \mathrm{OH}$ group is then used by the DNA polymerase to synthesize the new strand.

As DNA synthesis continues, the original DNA strands continue to unwind on each side of the bubble, forming a 
replication fork with two prongs. In bacteria, which have a single origin of replication on their circular chromosome, this process creates a "theta structure" (resembling the Greek letter theta: $\theta)$. In contrast, eukaryotes have longer linear chromosomes and initiate replication at multiple origins within these [7].

\section{Replication Fork}

The replication fork is a structure that forms within the nucleus during DNA replication. It is created by helicases, which break the hydrogen bonds holding the two DNA strands together. The resulting structure has two branching "prongs", each one made up of a single strand of DNA. These two strands serve as the template for the leading and lagging strands, which will be created as DNA polymerase matches complementary nucleotides to the templates; the templates may be properly referred to as the leading strand template and the lagging strand template.DNA is always synthesized in the $5^{\prime}$ to $3^{\prime}$ direction. Since the leading and lagging strand templates are oriented in opposite directions at the replication fork, a major issue is how to achieve synthesis of nascent (new) lagging strand DNA, whose direction of synthesis is opposite to the direction of the growing replication fork(Figure 2).

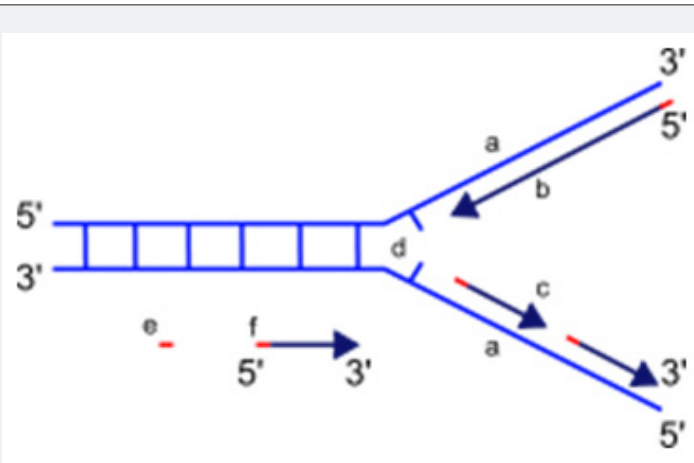

Figure 2: Replicate Fork.

Scheme of the replication fork a,Template; b,Leading strand; c,Lagging strand; d,Replication fork; e,Primer; f, Okazaki fragments

\section{Leading strand}

The leading strand is the strand of nascent DNA which is being synthesized in the same direction as the growing replication fork. A polymerase "reads" the leading strand template and adds complementary nucleotides to the nascent leading strand on a continuous basis. The polymerase involved in leading strand synthesis is DNA polymerase III (DNA Pol III) in prokaryotes and Pol $\delta$ in eukaryotes [8].Pol $\varepsilon$ can substitute for Pol $\delta$ in special circumstances [7].

Table 1: Enzyme and Function in DNA Replication.

\begin{tabular}{|c|c|}
\hline Enzyme & Function in DNA Replication \\
\hline DNA Helicase & Also known as helix destabilizing enzyme. Unwinds the DNA double helix at the Replication Fork. \\
\hline DNA Polymerase & $\begin{array}{c}\text { Builds a new duplex DNA strand by adding nucleotides in the 5' to 3' direction. Also performs proof-reading } \\
\text { and error correction. There exist many different types of DNA Polymerase, each of which performs different } \\
\text { functions in different types of cells. }\end{array}$ \\
\hline
\end{tabular}

\section{Lagging Strand}

The lagging strand is the strand of nascent DNA whose direction of synthesis is opposite to the direction of the growing replication fork. Because of its orientation, replication of the lagging strand is more complicated as compared to that of the leading strand.The lagging strand is synthesized in short, separated segments. On the lagging strand template, a primase "reads" the template DNA and initiates synthesis of a short complementary RNA primer. A DNA polymerase extends the primed segments, forming Okazaki fragments. The RNA primers are then removed and replaced with DNA, and the fragments of DNA are joined together by DNA ligase.

DNA polymerase III (in prokaryotes) or Pol $\delta$ (in eukaryotes) is responsible for extension of the primers added during replication of the lagging strand. Primer removal is performed by DNA polymerase I (in prokaryotes) and Pol $\delta$ (in eukaryotes). Eukaryotic primase is intrinsic to Pol $\alpha$ [9]. In eukaryotes, pol $\varepsilon$ helps with repair during DNA replication.

As helicase unwinds DNA at the replication fork, the DNA ahead is forced to rotate. This process results in a build-up of twists in the DNA a head [10]. This build-up forms a torsional resistance that would eventually halt the progress of the replication fork. Topoisomerases are enzymes that temporarily break the strands of DNA, relieving the tension caused by unwinding the two strands of the DNA helix; topoisomerases (including DNA gyrase) achieve this by adding negative supercoils to the DNA helix [11].Bare single-stranded DNA tends to fold back on itself forming secondary structures; these structures can interfere with the movement of DNA polymerase. To prevent this, single-strand binding proteins bind to the DNA until a second strand is synthesized, preventing secondary structure formation [12].

Clamp proteins form a sliding clamp around DNA, helping the DNA polymerase maintain contact with its template, thereby assisting with processivity. The inner face of the clamp enables DNA to be threaded through it. Once the polymerase reaches the end of the template or detects double-stranded DNA, the sliding clamp undergoes a conformational change that releases the DNA polymerase. Clamp-loading proteins are used to initially load the clamp, recognizing the junction between template and RNA primers [4].

\section{DNA Replication Proteins}

At the replication fork, many replication enzymes assemble on the DNA into a complex molecular machine called the replisome. The following is a list of major DNA replication enzymes that participate in the replisome [13] (Table 1). 
Current Trends in Biomedical Engineering \& Biosciences

\begin{tabular}{|c|c|}
\hline DNA clamp & A protein which prevents elongating DNA polymerases from dissociating from the DNA parent strand. \\
\hline Single-Strand Binding (SSB) Proteins & $\begin{array}{l}\text { Bind to ssDNA and prevent the DNA double helix from re-annealing after DNA helicase unwinds it, thus } \\
\text { maintaining the strand separation, and facilitating the synthesis of the nascent strand. }\end{array}$ \\
\hline Topoisomerase & Relaxes the DNA from its super-coiled nature. \\
\hline DNA Gyrase & Relieves strain of unwinding by DNA helicase; this is a specific type of topoisomerase \\
\hline DNA Ligase & Re-anneals the semi-conservative strands and joins Okazaki Fragments of the lagging strand. \\
\hline Primase & Provides a starting point of RNA (or DNA) for DNA polymerase to begin synthesis of the new DNA strand. \\
\hline Telomerase & $\begin{array}{l}\text { Lengthens telomeric DNA by adding repetitive nucleotide sequences to the ends of eukaryotic chromosomes. } \\
\text { This allows germ cells and stem cells to avoid the Hay flick limit on cell division[11]. }\end{array}$ \\
\hline
\end{tabular}

\section{Replication Machinery}

Replication machineries consist of factors involved in DNA replication and appearing on template ssDNAs. Replication machineries include primosomes also. The factors are replication enzymes; DNA polymerase, DNA helicases, DNA clamps and DNA topoisomerases, and replication proteins; e.g. single-stranded DNA binding proteins (SSB). In the replication machineries these components coordinate. In most of the bacteria, all of the factors involved in DNA replication are located on replication forks and the complexes stay on the forks during DNA replication. These replication machineries are called replisomes or DNA replicase systems; these terms mean originally a generic term for proteins located on replication forks. In eukaryotic and some bacterial cells, the replisomes are not formed.

Since replication machineries do not move relatively to template DNAs such as factories, they are called replication factory [1].In an alternative figure, DNA factories are similar to projectors and DNAs are like as cinematic films passing constantly into the projectors. In the replication factory model, after both DNA helicases for leading stands and lagging strands are loaded on the template DNAs, the helicases run along the DNAs into each other. The helicases remain associated for the remainder of replication process. Observed directly replication sites in budding yeast by monitoring green fluorescent protein (GFP)-tagged DNA polymerases $\alpha[14]$. They detected DNA replication of pairs of the tagged loci spaced apart symmetrically from a replication origin and found that the distance between the pairs decreased markedly by time. This finding suggests that the mechanism of DNA replication goes with DNA factories. Suggesting, couples of replication factories are loaded on replication origins and the factories associated with each other. Also, template DNAs move into the factories, which bring extrusion of the template ssDNAs and nascent DNAs. Peter's finding is the first direct evidence of replication factory model. By later researches, it is revealed that DNA helicases form dimers in many eukaryotic cells and bacterial replication machineries stay in single intranuclear location during DNA synthesis [15].

The replication factories perform disentanglement of sister chromatids. The disentanglement is essential to distribute the chromatids into daughter cells after DNA replication. Because sister chromatids after DNA replication hold each other by Cohesin rings, there is the only chance for the disentanglement in DNA replication. Fixing of replication machineries as replication factories can improve a success rate of DNA replication. If replication forks move freely in chromosomes, catenation of nuclei is aggravated and impedes mitotic segregation [14].

\section{Termination}

Eukaryotes initiate DNA replication at multiple points in the chromosome, so replication forks meet and terminate at many points in the chromosome; these are not known to be regulated in any particular way. Because eukaryotes have linear chromosomes, DNA replication is unable to reach the very end of the chromosomesbut ends at the telomere region of repetitive DNA close to the end. This shortens the telomere of the daughter DNA strand. Shortening of the telomeres is a normal process in somatic cells. As a result, cells can only divide a certain number of times before the DNA loss prevents further division. (This is known as the Hay flick limit.) Within the germ cell line, which passes DNA to the next generation, telomerase extends the repetitive sequences of the telomere region to prevent degradation. Telomerase can become mistakenly active in somatic cells, sometimes leading to cancer formation. Increased telomerase activity is one of the Hallmarks of cancer.

Termination requires that the progress of the DNA replication fork must stop or be blocked. Termination at a specific locus, when it occurs, involves the interaction between two components:

i. A termination site sequence in the DNA, and

ii. A protein which binds to this sequence to physically stop DNA replication.

In various bacterial species, this is named the DNA replication terminus site-binding protein, or Ter protein. Because bacteria have circular chromosomes, termination of replication occurs when the two replication forks meet each other on the opposite end of the parental chromosome. E. coli regulates this process through the use of termination sequences that, when bound by the Tus protein, enable only one direction of replication fork to pass through. As a result, the replication forks are constrained to always meet within the termination region of the chromosome [16].

\section{Eukaryotes}

Within eukaryotes, DNA replication is controlled within the context of the cell cycle. As the cell grows and divides, it progresses through stages in the cell cycle; DNA replication takes place during 
the $\mathrm{S}$ phase (synthesis phase). The progress of the eukaryotic cell through the cycle is controlled by cell cycle checkpoints. Progression through checkpoints is controlled through complex interactions between various proteins, including cyclins and cyclin-dependent kinases [17].

Unlike bacteria, eukaryotic DNA replicates in the confines of the nucleus [18]. The G1/S checkpoint (or restriction checkpoint) regulates whether eukaryotic cells enter the process of DNA replication and subsequent division. Cells that do not proceed through this checkpoint remain in the G0 stage and do not replicate their DNA. Replication of chloroplast and mitochondrial genomes occurs independently of the cell cycle, through the process of D-loop replication.

\section{Replication Focus}

In vertebrate cells, replication sites concentrate into positions called replication foci [11]. Replication sites can be detected by immune staining daughter strands and replication enzymes and monitoring GFP-tagged replication factors. By these methods it is found that replication foci of varying size and positions appear in $S$ phase of cell division and their number per nucleus is far smaller than the number of genomic replication forks.

Tracked GFP-tagged replication foci in budding yeast cells and revealed that replication origins move constantly in G1 and $S$ phase and the dynamics decreased significantly in S phase [14]. Traditionally, replication sites were fixed on spatial structure of chromosomes by nuclear matrix or lamins. The Heun's results denied the traditional concepts, budding yeasts don't have lamins, and support that replication origins self-assemble and form replication foci. By firing of replication origins, controlled spatially and temporally, the formation of replication foci is regulated [8] revealed that neighboring origins fire simultaneously in mammalian cells [14]. Spatial juxtaposition of replication sites brings clustering of replication forks. The clustering does rescue of stalled replication forks and favors normal progress of replication forks. Progress of replication forks is inhibited by many factors; collision with proteins or with complexes binding strongly on DNA, deficiency of dNTPs, nicks on template DNAs and so on. If replication forks stall and remaining sequences from the stalled forks are not replicated, daughter strands have nick obtained un-replicated sites. The un-replicated sites on one parent's strand hold the other strand together but not daughter strands. Therefore, resulting of sister chromatids cannot separate together and cannot divide into 2 daughter cells. When neighboring origins fire and a fork from one origin is stalled, fork from other origin access on an opposite direction of the stalled fork and duplicate the un-replicated sites. As other mechanism of the rescue there is application of dormant replication origins that excess origins don't fire in normal DNA replication.

\section{Bacteria}

Most bacteria do not go through a well-defined cell cycle but instead continuously copy their DNA; during rapid growth, this can result in the concurrent occurrence of multiple rounds of replication [19]. In E. coli, the best-characterized bacteria, DNA replication is regulated through several mechanisms, including: the hemi methylation and sequestering of the origin sequence, the ratio of adenosine triphosphate (ATP) to adenosine diphosphate (ADP), and the levels of protein DnaA. All these controls the binding of initiator proteins to the origin sequences. Because E. colimethylates GATC DNA sequences, DNA synthesis results in hemi methylated sequences. This hemi methylated DNA is recognized by the protein SeqA, which binds and sequesters the origin sequence; in addition, DnaA (required for initiation of replication) binds less well to hemi methylated DNA. As a result, newly replicated origins are prevented from immediately initiating another round of DNA replication [20].

ATP builds up when the cell is in a rich medium, triggering DNA replication once the cell has reached a specific size. ATP competes with ADP to bind to DnaA, and the DnaA-ATP complex is able to initiate replication. A certain number of DnaA proteins are also required for DNA replication. Each time the origin is copied, the number of binding sites for DnaA doubles, requiring the synthesis of more DnaA to enable another initiation of replication.

\section{Polymerase Chain Reaction}

Researchers commonly replicate DNA in vitro using the polymerase chain reaction (PCR). PCR uses a pair of primers to span a target region in template DNA, and then polymerizes partner strands in each direction from these primers using a thermo stable DNA polymerase. Repeating this process through multiple cycles amplifies the targeted DNA region. At the start of each cycle, the mixture of template and primers is heated, separating the newly synthesized molecule and template. Then, as the mixture cools, both of these become templates for annealing of new primers and the polymerase extends from these. As a result, the number of copies of the target region doubles each round, increasing exponentially [20].

\section{Summary and Conclusion}

DNA is a molecule that carries most of the genetic instructions used in the development, functioning and reproduction of all known living organisms and many viruses[21]. DNA is a nucleic acid, alongside proteins and carbohydrates. Nucleic acids composed of the three major macromolecules essential for all known forms of life. Each nucleotide is composed of nitrogen containing nucleobase cytosine $(C)$, guanine $(G)$, adenine $(A)$, and thymine (T) as well as a monosaccharide sugar called deoxyribose and a phosphate group. DNA replication is the process of producing two identical replicas from one original DNA molecule. DNA replication begins at specific locations, or origins of replication, in the genome[22].

DNA usually exists as a double-stranded structure, with both strands coiled together to form the characteristic double- helix. DNA strands have directionality and the different ends of a single strand are called the " 3 ' (three-prime) end" and the " 5 ' (five- 
prime) end". By convention, if the base sequence of a single strand of DNA is given, the left end of the sequence is the 5' end, while the right end of the sequence is the 3 ' end. The strands of the double helix are anti-parallel with one being5' to 3', and the opposite strand 3' to 5'[23,24]. DNA has several steps of replication. These are initiation, elongation and termination.Elongation also has replication fork, leading strand, lagging strand, DNA replication proteins, and replication machinery.

DNA replication within eukaryotes is controlled within the context of the cell cycle. In vertebrate cells, replication sites concentrate into positions called replication foci. Replication sites can be detected by immune staining daughter strands and replication enzymes and monitoring GFP-tagged replication factors[25]. Most bacteria do not go through a well-defined cell cycle but instead continuously copy their DNA; during rapid growth, this can result in the concurrent occurrence of multiple rounds of replication. Finally, DNA also replicate in vitro using the polymerase chain reaction (PCR) by uses a pair of primers to span a target region in template DNA, and then polymerizes partner strands in each direction from these primers using a thermo stable DNA polymerase. Repeating this process through multiple cycles amplifies the targeted DNA region.

\section{References}

1. Berg JM, Tymoczko JL, Stryer L, Clarke ND (2002) Chapter 27, Section 4: DNA Replication of Both Strands Proceeds Rapidly from Specific Start Site. In: Biochemistry. WH Freeman and Company, USA.

2. Alberts B, Johnson A, Lewis J, Raff M, Roberts K, et al. (2002) Intracellular Control of Cell-Cycle Events: S-Phase Cyclin-Cdk Complexes (S-Cdks) Initiate DNA Replication Once Per Cycle. In: Molecular Biology of the Cell, Garland Science, UK.

3. Alberts B, Johnson A, Lewis J, Raff M, Roberts K, et al. (2002) Chapter 5 : DNA Replication, Repair, and Recombination. In: Molecular Biology of the Cell Garland Science, UK. pp. 235-298.

4. Berg JM, Tymoczko JL, Stryer L, Clarke ND (2002) Chapter 27: DNA Replication, Recombination, and Repair. In: Bio-chemistry. WH Freeman and Company, USA.

5. Tobiason DM, Seifert HS (2006) The Obligate Human Pathogen, Neisseria gonorrhoeae, Is Polyploid. PLoS Biology 4(6): e185.

6. Johnson RE, Klassen R, Prakash L, Prakash S (2015) A Major Role of DNA Polymerase $\delta$ in Replication of Both the Leading and Lagging DNA Strands. Molecular Cell 59(2): 163-175.

7. Hansen, Barbara (2011) Biochemistry and Medical Genetics: Lecture Notes. Kaplan Medical. p. 21.

8. James D Watson, Tania A Baker, Stephen P Bell, Alexander Gann, Michael Levine, et al. (2008) Molecular Biology of the gene. Pearson Education: 237.
9. Elizabeth R Barry, Stephen D Bell (2006) DNA Replication in the Archaea. Microbiol Mol Biol Rev 70(4): 876-887.

10. Alberts B, Johnson A, Lewis J, Raff M, Roberts K, et al. (2002) DNA Replication Mechanisms: DNA Topoisomerases Prevent DNA Tangling During Replication. In: Molecular Biology of the Cell, Garland Science, UK.

11. Peter Meister, Angela Taddei, Susan M Gasser (2006) In and out of the Replication Factory. Cell 125(7): 1233-1235.

12. Alberts B, Johnson A, Lewis J, Raff M, Roberts K, et al. (2002) DNA Replication Mechanisms: Special Proteins Help to Open Up the DNA Double Helix in Front of the Replication Fork. In: Molecular Biology of the Cell, Garland Science, UK.

13. Griffiths AJF, Wessler SR, Lewontin RC, Carroll SB (2008) Introduction to Genetic Analysis. WH Freeman and Company, UK.

14. Lodish H, Berk A, Zipursky LS, Matsudaira P, Baltimore D, et al. (2000) General Features of Chromosomal Replication: Three Common Features of Replication Origins. In: Molecular Cell Biology. WH Freeman and Company, UK.

15. Huberman JA, Riggs AD (1968) On the mechanism of DNA replication in mammalian chromosomes. J Mol Biol 32(2): 327-341.

16. Brown TA (2002) Termination of replication. In: Genomes. BIOS Scientific Publishers.

17. Alberts B, Johnson A, Lewis J, Raff M, Roberts K, et al. (2002) Molecular Biology of the Cell. ( $4^{\text {th }}$ edn). Garland Science, UK. pp. 238-240.

18. Brown TA (2002) Genomes. (2nd edn). Oxford: Wiley-Liss, USA.

19. Slater S, Wold S, Lu M, Boye E, Skarstad K, et al. (1995) E. coli Seq A protein binds oriC in two different methyl-modulated reactions appropriate to its roles in DNA replication initiation and origin sequestration. Cell 82(6): 927-936.

20. Reece RJ, Maxwell A, Wang JC (1991) DNA Gyrase: Structure and Function. Crit Rev Biochem Mol Biol 26(3-4): 335-375.

21. Alberts B, Johnson A, Lewis J, Raff M, Roberts K, et al. (2002) Chapter 5: DNA Replication Mechanisms. In: Molecular Biology of the Cell, Garland Science, UK.

22. Aravind L, Leipe DD, Koonin EV (1998) Toprima conserved catalytic domain in type IA and II topoisomerases, DnaG-type primases, OLD family nucleases and RecR proteins. Nucleic acids research 26(18): 4205-4213.

23. Frick David, Richardson Charles (2001) DNA Primases. Annu Rev Biochem 70: 39-80.

24. Saiki RK, Gelfand DH, Stoffel S, Scharf SJ, Higuchi R, et al. (1988) Primer-directed enzymatic amplification of DNA with a thermostable DNA polymerase. Science 239(4839): 487-491.

25. Weigel C, Schmidt A, Rückert B, Lurz R, Messer W (1997) DnaA protein binding to individual DnaA boxes in the Escherichia coli replication origin, oriC. EMBO J 16(21): 6574-6783. 
This work is licensed under Creative Commons Attribution 4.0 License

DOI: 10.19080/CTBEB.2018.16.555942
Your next submission with Juniper Publishers will reach you the below assets

- Quality Editorial service

- Swift Peer Review

- Reprints availability

- E-prints Service

- Manuscript Podcast for convenient understanding

- Global attainment for your research

- Manuscript accessibility in different formats (Pdf, E-pub, Full Text, Audio)

- Unceasing customer service

Track the below URL for one-step submission https://juniperpublishers.com/online-submission.php 